\title{
Trend of Entamoeba histolytica infestation in Kolkata
}

\author{
Avik K Mukherjee ${ }^{1}$, Kaushik Das ${ }^{1}$, Mihir K Bhattacharya ${ }^{2}$, Tomoyoshi Nozaki ${ }^{3}$, Sandipan Ganguly ${ }^{1 *}$
}

\begin{abstract}
Background: Entamoeba histolytica infection is found almost all over the world and is highly endemic and a major cause of parasitic diarrhoea particularly in the developing countries.

Methods: A systemic surveillance was set up at the Infectious Disease hospital, Kolkata, India between November 2007 and October 2009 for understanding the trend of E. histolytica infection in Kolkata. Fecal samples were collected from diarrhoeal patients attending the hospital, under the surveillance system and processed for detection of E. histolytica.

Results: During the last two years about 2500 diarrhoeal samples were collected and screened for E. histolytica. About 3.6\% were positive for E. histolytica. As compared to the earlier years, E. histolytica infection was observed to be less amongst patients screened during the last two years. No seasonality was observed in Kolkata although in the neighboring tropical country Bangladesh, a typical seasonality of E. histolytica infection was noticed.

Conclusion: The study indicates that the detection rate of E. histolytica infection amongst diarrhoeal patients in Kolkata is decreasing during the last two years than that of Bangladesh.
\end{abstract}

\section{Background}

Amoebiasis caused by infection with $E$. histolytica occurs almost all over the world and is highly endemic especially in the developing countries. It is one of the major causes of dysentery/diarrhoea in Kolkata, India. According to our previous study (unpublished), detection of Entamoeba histolytica showed a marked seasonality, i.e. high peak during post-monsoon and post-winter seasons. According to the reports from other tropical countries, especially in Bangladesh, which is geographically closest to Kolkata, there is a typical pattern of detection of $E$. histolytic where E. histolytica usually shows its highest peaks in the wet season and gradually decreases with the arrival of dry season [1,2]. In a study in Bangladesh, it was shown that wet environment is not the only factor that affects the detection curve of E. histolytica, but anti-Carbohydrate Recognition Domain IgA level in the gut is another determining factor for its occurrence in a closed population [3]. However, even in that case E. histolytica detection

\footnotetext{
* Correspondence: sandipanganguly@gmail.com

'Department of Parasitology, National Institute of Cholera and Enteric Diseases, Indian Council of Medical Research, P33 CIT Road, Scheme XM, Kolkata - 700010, India

Full list of author information is available at the end of the article
}

followed a particular seasonality and trend. However in Kolkata the present scenario is different from other tropical regions.

\section{Study design and results}

For the last few years, we have been engaged with the surveillance study to determine the detection rate of common enteric parasites in Kolkata. During the last two years, about 2500 diarrhoeal stool samples were screened from hospitalized patients through a systematic sampling procedure as described previously [1] in collaboration with Infectious Diseases Hospital, Kolkata. In this surveillance, every fifth patient attending the Infectious diseases hospital from different parts of Kolkata with diarrhoea in randomly selected two days a week were included in the study. Fecal samples from these patients were collected and sent to the laboratory within half an hour of collection for detection of E. histolytica by microscopic examination using iodine wet mount method; PCR and antigen capture ELISA. The whole study was carried out from the month of November 2007 to October 2009 to include at least two seasonal cycles for better understanding of the seasonality. Although one of the major diarrhoea causing pathogen in Kolkata is Vibrio cholerae [4] 


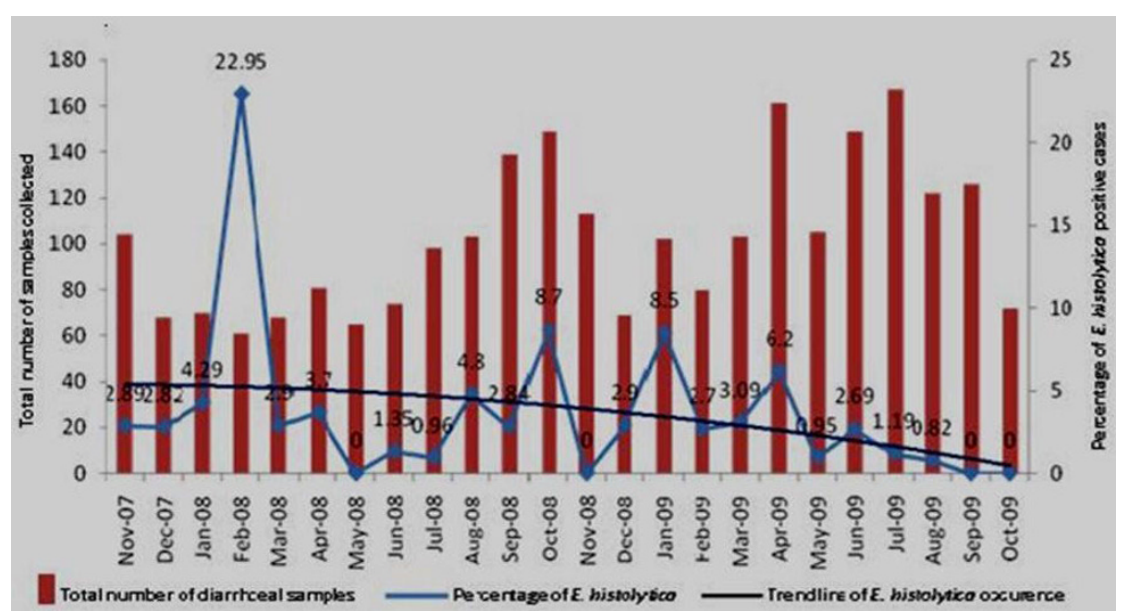

Figure 1 Month wise distribution of samples collected throughout the study period along with percentage of E. histolytica positive among them.

among all etiologies, E. histolytica (3.6\%) is one of the major diarrheagenic parasites among the parasitic etiologies of diarrhoea. It caused some sporadic diarrhea throughout the study period without any particular seasonality which is uncommon for a tropical area like Kolkata. Overall a decreasing trend of E. histolytica infection was also seen (Figure 1). Reasons behind this different nature of infection might be due to physical and environmental factors which could influence $E$. histolytica detection rate, such as choice of common antimicrobial drug, temperature, detection procedure of $E$. histolytica etc. But none of these changed strikingly in last two years that could affect $E$. histolytica detection rate. Indiscriminate use of anti-parasitic drugs like metronidazole and tinidazole could be attributed to the decreasing trend of detection of E. histolytica in Kolkata.

\section{Conclusion}

The present study demonstrates that there is no particular seasonality of occurrence of $E$. histolytica infection which is not typical of a tropical area like Kolkata. A different pattern which is almost unique in comparison to other tropical countries has been observed. Contribution of environmental and host factors as well as parasite genotypes are very important for the outcome of infection [2,5]. Although no physical or environmental factor behind this changing pattern of E. histolytica infestation in Kolkata has yet been reported, but it is certain that $E$. histolytica is showing a slow but obvious change in its seasonality and this might be a signal for a transition period of changing nature of infestation by this parasite in this part of the world. It is worth mentioning that impact of climate change might lead to such changes, although no such studies are available.

\section{Acknowledgements}

This study was supported by grants from

1. Japan Health Science Foundation and

2. Okayama University Program of Founding Research Centre for Emerging and Reemerging Infectious Disease, Ministry of Education, Culture, Sports, Science and Technology of Japan.

Authors also acknowledge the help and support given by Prof. Y. Takeda, Dr. G. B. Nair, Dr. Samiran Panda and Dr. K. Rajendran of NICED during preparation of this letter and Debarati Ganguly of Calcutta University for her careful proof reading and correction of English in the letter.

\section{Author details}

'Department of Parasitology, National Institute of Cholera and Enteric Diseases, Indian Council of Medical Research, P33 CIT Road, Scheme XM, Kolkata - 700010, India. ${ }^{2}$ Department of Clinical Medicine, National Institute of Cholera and Enteric Diseases, Indian Council of Medical Research, P33 CIT Road, Scheme XM, Kolkata - 700010, India. ${ }^{3}$ Division of Parasitology, National Institute of Infectious Diseases, Shinjuku, Tokyo, Japan.

Received: 1 September 2010 Accepted: 6 October 2010

Published: 6 October 2010

\section{References}

1. Mukherjee AK, Chowdhury P, Bhattacharya MK, Ghosh M, Rajendran K, Ganguly S: Hospital-based surveillance of enteric parasites in Kolkata. BMC Research Notes 2009, 2:110.

2. Ravdin Jl: Amebiasis; Series on Tropical Medicine. Science and Practice Imperial College Press 2000, 2:55.

3. Haque R, Mondal D, Duggal P, Kabir M, Roy S, Farr BM, Sack RB, Petri WA Jr: Entamoeba histolytica Infection in Children and Protection from Subsequent Amebiasis. Infect Immunity 2006, 904-909.

4. Nair Balakrish Gopinath, Ramamurthy Thandavarayan, Bhattacharya Kumar Mihir, Krishnan Triveni, Ganguly Sandipan, Saha Rani Dhira,

Rajendran Krishnan, Manna Byomkesh, Ghosh Mrinmoy, Okamoto Keinosuke, Takeda Yoshifumi: Emerging trends in the etiology of enteric pathogens as evidenced from an active surveillance of hospitalized diarrhoeal patients in Kolkata, India. Gut Pathogen 2010, 2:4.

5. Petri WA Jr, Mondal D, Peterson KM, Duggal P, Haque R: Association of malnutrition with amebiasis. Nutr Rev 2009, 67:S207.

doi:10.1186/1757-4749-2-12

Cite this article as: Mukherjee et al:: Trend of Entamoeba histolytica infestation in Kolkata. Gut Pathogens 2010 2:12. 